\title{
BMJ Open Virtual team-based care planning with older persons in formal care settings: a scoping review protocol
}

\author{
Harrison Gao (D) , ${ }^{1}$ Marie-Lee Yous (D) , ${ }^{2}$ Denise Connelly (D) , ${ }^{3}$ Lillian Hung (i) , \\ Anna Garnett (i) , ${ }^{5}$ Melissa Erin Hay (i) , ${ }^{3}$ Nancy Snobelen (D) , 6 \\ Samantha Salatino (D) ${ }^{7}$
}

To cite: Gao H, Yous M-L, Connelly D, et al. Virtual team-based care planning with older persons in formal care settings: a scoping review protocol. BMJ Open 2021;11:e054900. doi:10.1136/ bmjopen-2021-054900

- Prepublication history and additional supplemental material for this paper are available online. To view these files, please visit the journal online (http://dx.doi.org/10.1136/ bmjopen-2021-054900).

Received 25 June 2021 Accepted 21 October 2021

Check for updates

(C) Author(s) (or their employer(s)) 2021. Re-use permitted under CC BY-NC. No commercial re-use. See rights and permissions. Published by BMJ.

For numbered affiliations see end of article.

Correspondence to Dr Denise Connelly; dconnell@uwo.ca

\section{ABSTRACT}

Introduction COVID-19 has necessitated greater adoption of virtual care (eg, telephone (audio), videoconference) delivery models. Virtual care provides opportunities for innovative practice in care planning with older persons and meaningful family engagement by synchronously involving multiple care providers. Nevertheless, there remains a paucity of summarising evidence regarding virtual teambased care planning for older persons. The purpose of this scoping review is to summarise evidence on the utilisation of virtual team-based care planning for older persons in formal care settings. Specifically, (1) what has been reported in the literature on the impact or outcomes of virtual team-based care planning? (2) What are the facilitators and barriers to implementation?

Methods and analysis This scoping review will follow a rigorous and well-established methodology by the Joanna Briggs Institute, supplemented by the Arksey \& 0'Malley and Levac, Colquhoun, \& O'Brien frameworks. A three-step search strategy will be used to conduct a search on virtual team-based care planning for older persons in formal care settings. Keywords and index terms will be identified from an initial search in PubMed and AgeLine, and used to conduct the full search in the databases PubMed, EMBASE, CINAHL, AgeLine, Psycinfo and Scopus. Reference lists of included articles and grey literature retrieved through Google and Google Scholar will also be reviewed. Three researchers will screen titles and abstracts, and will conduct full-text review for inclusion. Extracted data will be mapped in a table.

Ethics and dissemination Research ethics approval is not required for data collection from publicly accessible information. Findings will be presented at conferences, submitted for open-access publication in a peer-reviewed journal and made accessible to multiple stakeholders. The scoping review will summarise the literature on virtual team-based care planning for the purpose of informing the implementation of a virtual PIECESTM intervention (Physical/Intellectual/Emotional health, Capabilities, Environment, and Social).

\section{INTRODUCTION}

The COVID-19 global pandemic has incited a greater adoption of virtual models of care delivery for healthcare services. The transition to virtual care including telephone (audio)
Strengths and limitations of this study

- This review will be timely and relevant to current and future COVID-19 responses and to support the development of a virtual delivery for PIECESTM (Physical/ Intellectual/Emotional health, Capabilities, Environment, and Social) intervention (a personcentred and family-centred care model for teambased practice).

- The review team includes a diverse team of older persons and family/care partners, clinicians, researchers and trainees in different phases of the scoping review.

- This review follows a rigorous methodology developed by the Joanna Briggs Institute in summarising evidence related to virtual team-based care planning.

- This scoping review will only include literature published in English.

- Literature on virtual care planning in formal care settings for older persons involving only a single healthcare discipline will be excluded from this review.

and videoconference clinical visits between older persons and providers was accelerated during the COVID-19 pandemic; however, the optimal role of virtual care is not clear. ${ }^{1}$ With the recent push to adopt virtual care, there is a need to ensure that person-centred and family-centred care continues to be at the forefront of care planning. Person-centred and family-centred care consists of full partnerships between older persons, families and healthcare providers to plan, provide and monitor health and social services addressing unique needs and goals. ${ }^{2-4} \mathrm{~A}$ team-based model of care delivery can help to ensure that the needs of older persons and families are being met. ${ }^{15}$ Team-based care models engage older persons and families as active participants throughout their journey of care, while supporting healthcare providers to deliver higher quality care and improve outcomes for older persons with complex care needs. ${ }^{5}$ 
Team-based care delivered virtually provides innovative opportunities to bring together family members and multiple clinicians in a single visit, thus improving older person-centred and family-centred care and interdisciplinary collaboration. ${ }^{1}$

Virtual care consists of remote interactions between older persons, families and healthcare teams using technology to support communication and information exchange. ${ }^{6}$ Virtual healthcare, such as telemedicine, has now been offered in many developed countries (eg, Canada, the USA, Norway and Australia) and in various types of formal care settings including primary care, hospitals, home and community care, and long-term care homes. ${ }^{17-9}$ With a global ageing population, virtual care may be necessary to support the growing number of older persons. Older persons require an integrated and comprehensive model of care delivery as many older persons have multiple chronic conditions, with $60 \%$ having at least two chronic conditions. ${ }^{10}$ There is a need for team-based care planning involving multiple disciplines in order to provide comprehensive care for older persons with multiple conditions. ${ }^{11}$ Another important aspect of the care planning process is ensuring that family members are able to be optimally engaged in meaningful ways since family involvement is mutually beneficial for patients and healthcare providers. ${ }^{4}$ However, family members often experience significant caregiver burden and need more support and information from healthcare teams. ${ }^{12}$

A team-based care delivery model with support for virtual care can also improve the well-being of clinicians. ${ }^{13} 14$ Silsand et al explored the experience of the Patient-Centred Team (PACT), an interdisciplinary healthcare team, supporting the transition from hospital to primary care services for older persons through videoconferencing during the COVID-19 pandemic. ${ }^{15}$ The virtual delivery of PACT was found to increase the efficient use of the expertise of clinicians, enhance collaboration between older persons and clinicians, and make meetings for older persons more convenient through reduced travel time. Other team-based virtual care delivery programmes for older persons have led to an increase in access to expert team follow-up with care, better support for organisations catering to older persons with more complex needs, enhanced communication between providers and greater accessibility of older person information for providers. ${ }^{7816}$ Some programmes, however, do not include older persons and/or family members during virtual care planning meetings. ${ }^{8}$ This is a barrier to care for older persons and families in taking on an active partnership role in care planning and voicing their concerns.

PIECES $^{\mathrm{TM}}$ (Physical/Intellectual/Emotional health, Capabilities, Environment, and Social) is a holistic clinical assessment framework intended for use by interdisciplinary teams to respond to the complex health needs of older persons. ${ }^{17}$ It was created as part of a long-term care initiative in Ontario, Canada, almost 25 years ago. ${ }^{17} 18$ PIECES is an acronym where the letters P-I-E reflect an individual's Physical, Intellectual and Emotional health. The letter $\mathrm{C}$ focuses on maximising the Capabilities of an individual to support quality of life. The final letters E-S integrate the living Environment of a person and the Social being encompassing a person's beliefs, culture and life story. ${ }^{17}$ PIECES has been implemented across Canada and in various settings, including acute care, long-term care, home and community care, complex continuing care and mental health settings. It is most often used to address responsive behaviours among older persons including words, sounds or actions expressed by persons with dementia to convey their physical and emotional needs such as pain, boredom, hunger or thirst. ${ }^{19}$ These behaviours can be exhibited through wandering, calling out, agitation and restlessness. ${ }^{20}$

To the best of our knowledge, there are only four published studies to date, which explored the implementation and evaluation of PIECES for older persons. ${ }^{21-24}$ In long-term care, PIECES has led to an increased ability of staff to recognise and address mental health challenges and social behaviours, more comprehensive assessments of older persons using a variety of existing tools, increased connections between long-term care homes and other resources, and improved family satisfaction with older person care. ${ }^{22}{ }^{23}$ In acute care hospital settings, PIECES improved interdisciplinary collaboration between different disciplines, promoted holistic assessment of older persons and encouraged problem-solving when addressing responsive behaviours. ${ }^{21}{ }^{24}$ Although PIECES has only been evaluated in populations diagnosed with dementia, cognitive impairment or mental health issues, our rationale for focusing on older persons more broadly was due to the limited body of literature on virtual teambased care planning. This scoping review also seeks to inform holistic virtual care planning, which involves looking at mental health and physical health issues that are often intertwined in older persons. ${ }^{8}$

Team-based care delivery of person-centred care for older persons is well documented. However, there are currently very few literature reviews exploring the virtual implementation and delivery of team-based care planning for older persons. ${ }^{11}{ }^{25}$ Inviting older persons and their families to participate in interdisciplinary team meetings to share their concerns and discuss their goals has also gained more attention in recent years. ${ }^{25}{ }^{26}$ In order to develop holistic person-centred approaches to care planning, it will be important to engage care partners at all stages of development and implementation given the pivotal role they have in supporting older persons (with care complexity). ${ }^{27}$ The summary of evidence on virtual team-based care planning will help inform the novel implementation of PIECES virtually, including which impacts and outcomes should be considered, and which strategies may enhance its success.

The purpose of this scoping review is to summarise and synthesise the current knowledge about virtual teambased care planning for older persons implemented in formal care settings. The specific research questions 
include the following: (1) What has been reported in the literature on the impact or outcomes of virtual teambased care planning? (2) What are the facilitators and barriers to implementation?

\section{METHODS}

This scoping review will follow the rigorous methodology devised by the Joanna Briggs Institute (JBI).$^{28}$ This methodology provides clear guidance on developing, conducting searches and analysing the body of relevant literature. ${ }^{28}$ It is also used by published scoping reviews. ${ }^{29}$ The five-stage framework developed by Arksey \& O'Malley and enhanced by Levac, Colquhoun, \& O'Brien helped establish the JBI methodology and will supplement our scoping review methodology. ${ }^{30} 31$ The five-stage framework guided the development of the research questions, inclusion/exclusion criteria and data extraction tools, and will provide additional guidelines on conducting the scoping review (ie, identifying, selecting and summarising evidence). The Preferred Reporting Items for Systematic Reviews and Meta-Analyses Extension for Scoping Reviews checklist (PRISMA-ScR), complementary with the JBI methodology, will help structure the proposed scoping review. ${ }^{32}$

A scoping review was chosen for this study with the aim of summarising key findings from the research literature rather than providing a critical appraisal of relevant studies or drawing a conclusion to a specific question as in a systematic review. ${ }^{28}{ }^{33}$ Summarising the existing literature within a scoping review may help identify research gaps for future research and inform development and application of interventions to address the identified $\operatorname{gap}(\mathrm{s}) .{ }^{35}$

A diverse team of researchers, clinicians, trainees, older persons and family care partners will be involved in different phases of the scoping review. This team is led by researchers and clinicians with expertise in supporting older persons and families in long-term care, acute care, and home and community care. Two trainees (HG, M-LY) will conduct searches for relevant literature under the guidance and mentorship of an academic professor (DC). Articles will be reviewed afterwards by HG, M-LY and a postdoctoral fellow (MEH) based on the inclusion criteria. A group of older person partners will be invited to review themes and provide their lived experience related to the themes. All members of the research team will be engaged in the analysis through regular team meetings where emerging themes will be developed and revised through reasoned discussion until consensus has been reached. The entire research team will review, revise and approve the final manuscript.

\section{Inclusion criteria}

As per the JBI methods, the inclusion criteria for the scoping review were framed using the PCC (Participants, Concept and Context) mnemonic. ${ }^{28}$ In terms of Participants, studies involving older persons (aged 60 or older) will be included in this review as older persons have complex care needs and a high prevalence of multiple chronic conditions. ${ }^{10}$ Older persons are defined as aged 60 or older to increase the number of available articles to inform the review, and to align with age groups in published scoping reviews and systematic reviews. ${ }^{28} 36$ Also, relevant studies involving interdisciplinary healthcare teams (ie, teams involving more than a single discipline), family/friend care partners, and/or active older person/family participation in virtual care planning will be included. Care partner and family level of involvement in studies will be reported and discussed.

The core Concepts of this review will be the implementation and evaluation of virtual team-based care planning for the purpose of informing holistic, interdisciplinary, person-centred and family-centred virtual care planning including a virtual delivery model of PIECES. The virtual team-based care planning review will focus on care, which involves engaging older persons, family members and multiple clinicians in a group meeting. In terms of Context, formal care settings refer to primary care, hospitals, home and community care, and long-term care. Other settings involving formal healthcare providers (eg, trained staff, nurses, personal support workers, physicians, allied health professionals) may be considered a formal care setting.

\section{Types of evidence and information sources}

The inclusion criteria for types of evidence and information sources are as follows. Published studies and grey literature in English from 1980 to present will be included in this review. The search for published studies will be conducted in the databases: PubMed, EMBASE, CINAHL, AgeLine, PsycInfo and Scopus. All forms of published study designs will be considered including reviews, quantitative, qualitative and mixed methods. Grey literature (eg, newsletters, reports, articles, guidelines, theses) retrieved from Google will be considered for inclusion to ensure that we cover the current breadth of knowledge. Published reports, unpublished reports and related articles will be searched using Google Scholar. We will also review an evidence list provided on the official PIECES Canada Learning Website when providing context on the PIECES ${ }^{\mathrm{TM}}$ intervention. We will contact PIECES experts and members of PIECES Canada to inquire about relevant literature.

\section{Search strategy}

The JBI Methodology recommends a three-step search strategy. We will be using the JBI three-step search strategy to conduct a search on the core concept of 'virtual team-based care planning for older persons'. The first step involved an initial limited search of at least two appropriate online databases, which was reviewed by a university librarian with expertise in conducting reviews, followed by an analysis of text words in the titles and abstracts of relevant articles. Keywords and index terms used to describe the articles were also identified. We 
conducted our initial limited search on 'virtual teambased care planning for older persons' in PubMed and AgeLine. These two databases were selected as they are more likely to provide relevant information related to the topic. See online supplemental file 1 for the search terms used in the initial limited search and three step search strategy.

In the second step, identified keywords and index terms from the initial limited search will be used to conduct a second search in all selected databases. Articles will be screened against inclusion criteria described using the PCC mnemonic. Relevant articles will include older persons in a formal care setting, involve a virtual setting (audio, video, mobile application), and involve the implementation and evaluation of team-based care planning. We define a team as encompassing more than one type of clinician in care planning for older persons. Clinician types include but are not limited to physicians, geriatricians, specialists, pharmacists, social workers, nurses, physical therapists, occupational therapists and others.

In the third step, the reference lists of all articles and reports selected to be included in the review will be hand searched for additional studies. Unless the outcomes are determined to be different after screening, only the most recent report on a study will be included in the review. Ongoing consultation with a university librarian experienced with systematic and scoping reviews will be used to refine the search strategy. As per the JBI guidelines, a full example search strategy conducted in PubMed is included. See online supplemental file 1 for the final search terms that will be used in the database and grey literature search, and the full three-step search strategy.

\section{Study selection}

The review management software Covidence will be used for study selection and reviewing results. ${ }^{37}$ All identified citations will be exported and uploaded to Covidence, where duplicates will be removed. Grey literature results will be downloaded from Google, then uploaded and screened through the same process. First, titles and abstracts will be screened by two independent reviewers (HG, M-LY) for relevancy. Then, full-text articles will be retrieved for relevant articles and assessed in detail against inclusion/exclusion criteria by all three reviewers, independently (HG, M-LY, MEH). At this stage, reasons for exclusion will be recorded and reported for any full-text studies that do not meet inclusion criteria. Any conflicts arising about inclusion will be resolved through discussion. The study selection criteria will be pilot tested on a random sample of 25 titles/abstracts and full-text articles prior to embarking on screening to refine study selection by the team of reviewers. As suggested by the JBI methodology, screening will only begin when $75 \%$ agreement is achieved. Final results of the search will be reported through a PRISMA-SCR flow diagram in the scoping review report.

\section{Data extraction}

Data will be extracted from articles and reports by charting results using a data extraction tool/table. NVivo V.12.0, a data management software, will be used to assist in summarising themes during data extraction and qualitative data analysis. ${ }^{38}$ Three researchers (HG, M-LY, $\mathrm{MEH}$ ) will extract relevant logical and descriptive data on author, year, country, type of evidence source, purpose, population/participants, setting/context, study design, intervention, outcome measures and relevant findings to the research questions. Some examples of outcomes of interest include feasibility, acceptability and reliability of the virtual intervention, older person and/or family member satisfaction with virtual care, physical and mental health of older persons and/or family members, and interdisciplinary team collaboration and communication. The data extraction tool will be revised as necessary during data extraction and changes will be reported in the scoping review report. A draft data extraction tool is provided (see online supplemental file 2). In accordance with the JBI methodology, the extraction tool will be pilot tested on three full-text articles and mapped results will be compared. Study and methodological quality will not be assessed as the goal of this scoping review is not to provide a critical appraisal of evidence. ${ }^{34}$

\section{Data synthesis}

Extracted data will be mapped in a literature table and accompanied by a narrative summary that will connect results to the study objective and research questions. Relevant findings will be used to inform the future research implementation of a virtual model of PIECES and evaluation of holistic, interdisciplinary, person-centred and family-centred virtual care planning.

\section{Patient and public involvement}

Clinicians (registered practical nurses (RPNs)) and executives who are currently working on implementing a novel virtual PIECES intervention in long-term care were involved in reviewing and providing feedback on the design of this protocol. Older persons, family members, clinicians and managers involved in this study were recruited from two long-term care home partners in Southern Ontario, Canada, through the Healthcare Excellence Canada (formerly Canadian Foundation for Healthcare Research and Canadian Patient Safety Institute) - Long-Term Care+, Acting on Pandemic Learning Together and Implementation Science Team, Strengthening Pandemic Preparedness in Long-Term Care initiatives. These older persons and family/care partners have experience seeking care beyond long-term care such as primary care, acute care, and home and community care. They will join the team of researchers in naming the themes and discussing the data supporting the themes. Older persons and family/care partners will be engaged in one or two ZOOM video-conferencing meetings to complete these tasks and provide insights from their lived 
experiences to shape discussion of the themes in the manuscript.

\section{Ethics and dissemination}

For the scoping review, research ethics approval and consent to participate will not be required. The proposed review will provide a transparent report based on rigorous and comprehensive scoping review methodologies. Findings of this scoping review will be used to support the basis of a subsequent study which has received ethics approval from University Research Ethics Boards (\#118629 and \#H21-01428). The proposed scoping review will be completed and submitted for publication in an open-access and peer-reviewed journal. Results will be made accessible to the public, policy makers, clinicians, healthcare managers and researchers, among others, and presented at relevant conferences. The findings will be used to guide the Healthcare Excellence Canada Implementation Science Team's project: 'COVID-19: Implementation of virtual PIECES ${ }^{\mathrm{TM}}$ for LTC resident care planning with family to build and sustain team collaboration and workforce resilience'. This project involves a pan-Canadian team of researchers and professional organisations such as PIECES Canada, Registered Practical Nurses Association of Ontario (WeRPN), and Strategy for Patient-Oriented Research (SPOR) Ontario Support Unit to implement a novel virtual PIECES intervention in long-term care homes. Various stakeholders are engaged in this research, including older persons and family, registered practical nurses and other healthcare providers.

\section{DISCUSSION}

The expected result of this scoping review will be a comprehensive summary of evidence regarding virtual team-based care planning for older persons receiving care in formal care settings. The summary of evidence about virtual team-based care planning will provide an in-depth understanding of the impact of virtual-based care planning on improving person-centred and family-centred care with older persons and families as well as interdisciplinary collaboration among providers in formal care settings. Findings from this scoping review may be used to inform a virtual adaptation of the PIECES intervention in supporting care planning as a holistic, interdisciplinary approach for older persons with complex care needs and their families in long-term care.

Previous work reviewing videoconferencing for older persons was related to healthcare provision, that is, clinical assessment, management, diagnosis, support, but did not address virtual team-based care planning. ${ }^{39}$ In their scoping review, Newbould et al reported that one of the most common reasons for long-term care homes to consider videoconferencing was to increase the satisfaction of healthcare providers in delivering care for older persons. ${ }^{39}$ Teamwork leads to higher role engagement, which, in turn, leads to greater job satisfaction and can mitigate the effects of work demands and burnout. ${ }^{40}$ Despite the advantages of virtual care, there are known barriers in using videoconferencing for older persons. Barriers include lack of access to equipment and ability to independently use technological devices, and sensory impairments leading to communication challenges. ${ }^{941} \mathrm{It}$ is important to consider whether older persons are comfortable with using video technology and/or have disabilities that would limit the optimal use of video conferencing. ${ }^{42}$ There are also barriers of access and equity issues related to the social determinants of health (eg, socioeconomic factors, geography, culture/language, gender). ${ }^{9}$ Virtual care barriers can be addressed with support from family, friends or clinicians.

Team-based care planning is, therefore, a critical concept to translate into virtual interventions as it can improve older person outcomes and the well-being of healthcare providers. ${ }^{13}$ Supporting older persons, their families and the workforce is likely to contribute to greater resilience when faced with challenges such as pandemics. The success of healthcare teams is dependent on opportunities for regular team meetings, team coordination, leadership and open communication. ${ }^{13}$ Videoconferencing is an effective alternative to in-person meetings offering tailored support for persons with complex chronic care needs by enhancing access to multiple providers within a fragmented care system. ${ }^{15} 43$ Compared with in-person care, virtual care offers greater opportunities for efficient use of resources by facilitating meeting convenience for older persons and families, improving the communication of information among providers and involving multiple providers in coordinating care. ${ }^{15}$

The proposed scoping review will provide an updated summary in the literature about the use of virtual teambased interventions with older persons in formal care settings. As seen during the initial phases of the COVID-19 pandemic, older persons and family care partners were the most impacted by the pandemic with reduced opportunities to meet with providers. Older persons were also at higher risks of contracting the COVID-19 virus compared with the general population. A virtual model of teambased care delivery may ensure that older persons seeking services from formal care settings continue to receive high-quality assessments while adhering to infection control practices, allowing for better care coordination of a synchronous care session involving multiple healthcare providers and families, and instilling confidence among families that all providers are well informed of proposed interventions/treatments. ${ }^{1}$

COVID-19 has led many clinicians to re-evaluate their ways of delivering care for older persons and incorporate virtual care within their practice in some form. In planning for the post-pandemic future, patient preferences for in-person care and technology access should influence blended care delivery models. ${ }^{44}$ Face-to-face healthcare encounters can promote social engagement, may provide opportunities to express concerns and thoughts more freely, and could help older persons, especially those with 
language barriers, to better understand medical information. 4445

Virtual geriatric care can provide equity of access to specialised care and enable organisations including those in rural and remote settings to provide high quality care for older persons with complex issues. ${ }^{8}$ It is important to recognise that virtual care may perpetuate existing equity issues due to lack of access to and affordability of reliable internet and video-compatible devices. ${ }^{46}$ To ensure equitable access to virtual care, organisations should provide broadband internet access, education in digital literacy and devices to individuals who lack access. ${ }^{46}$ A need exists for a virtual intervention that will equip organisations and healthcare providers to provide holistic healthcare for older Canadian adults living in long-term care and other formal care settings.

Informed by the results of this scoping review, the PIECES research intervention will be the first to explore the implementation process of virtual PIECES to increase the meaningful engagement of multiple stakeholders (older persons living in long-term care, family and clinicians) in virtual team-based care planning. This research will seek to engage family care partners and older persons, build resilience in RPNs, improve long-term care preparedness for future outbreaks, and provide a plan for the adaption, implementation and sustainment of virtual PIECES. The proposed scoping review will provide a comprehensive summary of the current breadth of knowledge and provide new directions for further research: systematic reviews; research on PIECES; or research on virtual holistic care planning. It will provide a greater understanding of further adaptations required for implementation in long-term care homes including modifications to current models of care delivery, coaching and mentoring.

The scoping review will be limited to literature published in English, formal care settings and older person participants. Our search will not include literature on virtual care planning for older persons that involves only one single discipline (ie, not interdisciplinary). This review will provide timely and relevant findings to the current COVID-19 pandemic response, the development and exploration of a novel virtual PIECES intervention, and the push for both virtual and person/family-centred care for the growing older person population.

\section{Author affiliations}

${ }^{1}$ Faculty of Science, University of Western Ontario, London, Ontario, Canada

${ }^{2}$ McMaster University School of Nursing, Hamilton, Ontario, Canada

${ }^{3}$ School of Physical Therapy, Western University, London, Ontario, Canada

${ }^{4}$ School of Nursing, The University of British Columbia, Vancouver, British Columbia,

Canada

${ }^{5}$ Arthur Labatt Family School of Nursing, Western University, London, Ontario,

Canada

${ }^{6}$ Registered Practical Nurses Association of Ontario, Mississauga, Ontario, Canada

${ }^{7}$ Markham Stouffville Hospital, Markham, Ontario, Canada
Twitter Harrison Gao @harrisongao_, Marie-Lee Yous @MarieLeeYous, Lillian Hung @nurselillian and Anna Garnett @AnnaGarnett6

Acknowledgements The authors wish to thank the Registered Practical Nurses Association of Ontario, P.I.E.C.E.S. Canada, the Ontario SPOR Support Unit and the Canadian Gerontological Nursing Association. We are grateful for the older persons, family partners, clinicians and long-term care home executives on our team and their involvement in the scoping review. We are thankful for the support of the longterm care homes, Vision 74 LTC and APANS Health Services-Copper Terrace homes. We also want to thank Dr Robert Maunder and Eddy Nason from SPOR Support Unit for their involvement.

Contributors $\mathrm{HG}$ and M-LY drafted and developed the protocol manuscript. DC, $\mathrm{LH}, \mathrm{MEH}$ and $\mathrm{AG}$ critically reviewed the protocol manuscript, helped refine research questions and provided guidance for the scoping review objective and methods. HG, M-LY, DC, LH, AG, MEH, NS and SS made meaningful contributions to the conceptualisation, design and development of the study protocol through regular team meetings and collaborated on manuscript editing and revisions. All authors reviewed and approved the final version of the manuscript.

Funding The LTC+: Acting on Pandemic Learning Together funding competition (\#: SL5-174744) was managed and administered by Healthcare Excellence Canada, in partnership with the Canadian Institutes of Health Research's Institute of Health Services and Policy Research (IHSPR), Institute of Aging (IA), Institute of Gender and Health (IGH), Institute of Infection and Immunity (III), Institute of Circulatory and Respiratory Health (ICRH), Institute of Population and Public Health (IPPH), Institute of Musculoskeletal Health and Arthritis (IMHA), the Saskatchewan Health Research Foundation (SHRF), the New Brunswick Health Research Foundation (NBHRF), the Centre for Aging + Brain Health Innovation (CABHI), powered by Baycrest, and the Michael Smith Foundation for Health Research (MSFHR).

Competing interests None declared.

Patient consent for publication Not applicable.

Provenance and peer review Not commissioned; externally peer reviewed.

Supplemental material This content has been supplied by the author(s). It has not been vetted by BMJ Publishing Group Limited (BMJ) and may not have been peer-reviewed. Any opinions or recommendations discussed are solely those of the author(s) and are not endorsed by BMJ. BMJ disclaims all liability and responsibility arising from any reliance placed on the content. Where the content includes any translated material, BMJ does not warrant the accuracy and reliability of the translations (including but not limited to local regulations, clinical guidelines, terminology, drug names and drug dosages), and is not responsible for any error and/or omissions arising from translation and adaptation or otherwise.

Open access This is an open access article distributed in accordance with the Creative Commons Attribution Non Commercial (CC BY-NC 4.0) license, which permits others to distribute, remix, adapt, build upon this work non-commercially, and license their derivative works on different terms, provided the original work is properly cited, appropriate credit is given, any changes made indicated, and the use is non-commercial. See: http://creativecommons.org/licenses/by-nc/4.0/.

ORCID iDs

Harrison Gao http://orcid.org/0000-0003-0406-2041

Marie-Lee Yous http://orcid.org/0000-0002-4271-0401

Denise Connelly http://orcid.org/0000-0001-8138-1746

Lillian Hung http://orcid.org/0000-0002-7916-2939

Anna Garnett http://orcid.org/0000-0001-7111-8602

Melissa Erin Hay http://orcid.org/0000-0002-8675-5441

Nancy Snobelen http://orcid.org/0000-0002-5416-3740

Samantha Salatino http://orcid.org/0000-0003-3543-1233

\section{REFERENCES}

1 Sinsky CA, Jerzak JT, Hopkins KD. Telemedicine and team-based care: the perils and the promise. Mayo Clin Proc 2021;96:429-37.

2 Ball T. Disruptive innovation: patient/family-focused care. Managing Change 2010;90:421-56.

3 Epstein RM, Street RL. The values and value of patient-centered care. Ann Fam Med 2011;9:100-3.

4 Johnson BH, Abraham MR. Partnering with patients, residents, and families: a resource for leaders of hospitals, ambulatory care settings, and long-term care communities. Institute for Patient-and FamilyCentered Care, 2012. 
5 Ritchie C, Andersen R, Eng J, et al. Implementation of an interdisciplinary, team-based complex care support health care model at an academic medical center: impact on health care utilization and quality of life. PLoS One 2016;11:e0148096.

6 Shaw J, Jamieson T, Agarwal P, et al. Virtual care policy recommendations for patient-centred primary care: findings of a consensus policy dialogue using a nominal group technique. $J$ Telemed Telecare 2018;24:608-15.

7 Emery EE, Lapidos S, Eisenstein AR, et al. The BRIGHTEN program: implementation and evaluation of a program to bridge resources of an interdisciplinary geriatric health team via electronic networking. Gerontologist 2012;52:857-65.

8 Gray LC, Fatehi F, Martin-Khan M, et al. Telemedicine for specialist geriatric care in small rural hospitals: preliminary data. J Am Geriatr Soc 2016:64:1347-51.

9 Yi JS, Pittman CA, Price CL, et al. Telemedicine and dementia care: a systematic review of barriers and facilitators. J Am Med Dir Assoc 2021;22:1396-402.

10 National Institute on Aging. Supporting older patients with chronic conditions, 2017. Available: https://www.nia.nih.gov/health/ supporting-older-patients-chronic-conditions [Accessed May 2021].

11 Johansson G, Eklund K, Gosman-Hedström G. Multidisciplinary team, working with elderly persons living in the community: a systematic literature review. Scand J Occup Ther 2010;17:101-16.

12 Mcllfatrick S, Doherty LC, Murphy M, et al. 'The importance of planning for the future': burden and unmet needs of caregivers' in advanced heart failure: a mixed methods study. Palliat Med 2018;32:881-90.

13 Smith CD, Balatbat C, Corbridge S, et al. Implementing optimal team-based care to reduce clinician burnout. NAM Perspect 2018;8.

14 Welp A, Manser T, teamwork I. Clinician occupational well-being and patient safety-development of a conceptual framework based on a systematic review. BMC Health Serv Res 2016;16:1-44.

15 Silsand L, Severinsen G-H, Berntsen G. Preservation of PersonCentered care through videoconferencing for patient follow-up during the COVID-19 pandemic: case study of a multidisciplinary care team. JMIR Form Res 2021;5:e25220.

16 Farris G, Sircar M, Bortinger J. Extension for community healthcare outcomes - care transitions: enhancing geriatric care transitions through a multidisciplinary videoconference. American Geriatrics Society 2017;65:598-602.

17 Hamilton P, LeClair JK, Collins J, et al. PIECES Resource Guide: Guiding Collaborative Engagement, Shared Assessment and Supportive Care (7 Edition) - A Learning Resource for Health Care Practitioners Suporting Older Adults Living with Complexity, (). Pieces Canada: Sportswood Printing, 2020. www.pieceslearning. com

18 Ontario Ministry of Health and Long-Term Care. Ontario's Strategy for Alzheimer Disease and Related Dementias: Preparing for Our Future. Seniors' Secretariat, 1999.

19 Dupuis SL, Wiersma E, Loiselle L. Pathologizing behavior: meanings of behaviors in dementia care. J Aging Stud 2012;26:162-73.

20 Alzheimer Society of Canada. Responsive and reactive behaviour, 2021. Available: https://alzheimer.ca/en/help-support/im-caringperson-living-dementia/understanding-symptoms/responsivereactive-behaviours?gclid=CjOKCQjw4cOEBhDMARIsAA3XDRgubkOq9pn34Gk9r-FqOn1Xuo3WnSXEBVZOnm1Tx69BHUcYGHDjLs aAmG1EALw_wcB [Accessed May 2021].

21 Hung L, Lee PA, Au-Yeung AT, et al. Adopting a clinical assessment framework in older adult mental health. $J$ Psychosoc Nurs Ment Health Serv 2016;54:26-31.

22 McAiney CA, Stolee P, Hillier LM, et al. Evaluation of the sustained implementation of a mental health learning initiative in long-term care. Int Psychogeriatr 2007;19:842-58.

23 Stolee P, McAiney CA, Hillier LM, et al. Sustained transfer of knowledge to practice in long-term care: facilitators and barriers of a mental health learning initiative. Gerontol Geriatr Educ 2009;30:1-20.
24 Yous M-L, Ploeg J, Kaasalainen S, et al. Nurses' experiences in caring for older adults with responsive behaviors of dementia in acute care. SAGE Open Nurs 2019;5:237796081983412.

25 Giosa JL, Holyoke P, Stolee P. Let's get real about Person- and Family-Centred geriatric home care: a realist synthesis. Can J Aging 2019;38:449-67.

26 Clay AM, Parsh B. Patient- and Family-Centered care: it's not just for pediatrics anymore. AMA J Ethics 2016;18:40-4.

27 Elliott J, McNeil H, Ashbourne J, et al. Engaging older adults in health care decision-making: a realist synthesis. Patient 2016;9:383-93.

28 Peters MDJ, Godfrey C, Mclnerney P. Chapter 11: Scoping Reviews (2020 version). In: Aromataris E, Munn Z, eds. JBI Manual for Evidence Synthesis. JBI, 2020. https://synthesismanual.jbi.global

29 Hung L, Chow B, Shadarevian J. Using touchscreen tablets to support social connections and reduce responsive behaviours among people with dementia in care settings: a scoping review. Dementia (London), 2021: 20. 1124-43.

30 Arksey H, O'Malley L. Scoping studies: towards a methodological framework. Int J Soc Res Methodol 2005;8:19-32.

31 Levac D, Colquhoun H, O'Brien KK. Scoping studies: advancing the methodology. Implementation Sci 2010;5:1-9.

32 Tricco AC, Lillie E, Zarin W, et al. PRISMA extension for scoping reviews (PRISMA-ScR): checklist and explanation. Ann Intern Med 2018;169:467-73.

33 Armstrong R, Hall BJ, Doyle J, et al. 'Scoping the scope' of a cochrane review. J Public Health 2011;33:147-50.

34 Munn Z, Peters MDJ, Stern C, et al. Systematic review or scoping review? guidance for authors when choosing between a systematic or scoping review approach. BMC Med Res Methodol 2018;18:143.

35 Samnani SS, Vaska M, Ahmed S, et al. Review typology: the basic types of reviews for synthesizing evidence for the purpose of knowledge translation. J Coll Physicians Surg Pak 2017;27:635-41.

36 Franco MR, Tong A, Howard K, et al. Older people's perspectives on participation in physical activity: a systematic review and thematic synthesis of qualitative literature. Br J Sports Med 2015;49:1268-76.

37 Innovation VH. Covidence systematic review software, Melbourne, Australia. Available: https://www.covidence.org/ [Accessed Jun 2021].

38 NVivo. NVivo qualitative data analysis software. QSR International Pty Ltd. Version 12, 2018.

39 Newbould L, Mountain G, Hawley MS, et al. Videoconferencing for health care provision for older adults in care homes: a review of the research evidence. Int J Telemed Appl 2017;2017:1-7.

40 Mijakoski D, Karadzinska-Bislimovska J, Milosevic M. Differences in burnout, work demands and team work between Croatian and Macedonian Hospital nurses. Cogn Brain Behav 2015;19:179-200.

41 Appleman ER, O'Connor MK, Rockefeller W, et al. Using video telehealth to deliver patient-centered collaborative care: the GIMPACT pilot. Clin Gerontol 2020;31:1-10.

42 Wong A, Bhyat R, Srivastava S, et al. Patient care during the COVID-19 pandemic: use of virtual care. J Med Internet Res 2021;23:e20621.

43 Davis SM, Jones A, Jaynes ME, et al. Designing a multifaceted telehealth intervention for a rural population using a model for developing complex interventions in nursing. BMC Nurs 2020;19:9.

44 Hyde AM, Watt M, Carbonneau M, et al. Understanding preferences toward virtual care: a Pre-COVID mixed methods study exploring the perspectives of patients with chronic liver disease. Telemed J E Health 2021. doi:10.1089/tmj.2021.0099. [Epub ahead of print: 03 Jun 2021].

45 Dassieu L, Sourial N. Tailoring interventions for social isolation among older persons during the COVID-19 pandemic: challenges and pathways to healthcare equity. Int J Equity Health 2021;20:1-4. doi:10.1186/s12939-020-01360-8

46 Kaplan B, Access KB. Access, equity, and neutral space: telehealth beyond the pandemic. Ann Fam Med 2021;19:75-8. 